\title{
Predictive implications of nucleoside metabolizing enzymes in premenopausal women with node-positive primary breast cancer who were randomly assigned to receive tamoxifen alone or tamoxifen plus tegafur-uracil as adjuvant therapy
}

\author{
MASAKAZU TOI ${ }^{1}$, TADASHI IKEDA ${ }^{2}$, FUTOSHI AKIYAMA ${ }^{3}$, MASAFUMI KUROSUMI ${ }^{4}$, \\ HITOSHI TSUDA ${ }^{5}$, GOI SAKAMOTO ${ }^{6}$ and OSAHIKO ABE $^{7}$ \\ ${ }^{1}$ Department of Surgery (Breast Surgery), Graduate School of Medicine, Kyoto University, Kyoto; \\ ${ }^{2}$ Department of Surgery, Teikyo University School of Medicine, ${ }^{3}$ Department of Breast Pathology, Cancer Institute \\ of Japanese Foundation for Cancer Research, Tokyo; ${ }^{4}$ Department of Pathology, Saitama Cancer Center Hospital, \\ Ina-machi, ${ }^{5}$ Department of Pathology II, National Defense Medical College, Tokorozawa, Saitama; \\ ${ }^{6}$ Sakamoto Memorial Clinic, Academy of Breast Pathology, ${ }^{7}$ St. Luke's Hospital, Tokyo, Japan
}

Received May 7, 2007; Accepted June 29, 2007

\begin{abstract}
Recent studies have demonstrated that tegafur-uracil (UFT) is useful for the adjuvant treatment of various types of cancers. To determine whether nucleoside metabolizing enzymes could be used to predict the response to UFT treatment in women with primary breast cancer, we retrospectively analyzed archived tumor tissue samples obtained from the 3rd Adjuvant Chemo-Endocrine Therapy for Breast Cancer (ACETBC) study, in which adjuvant treatment with tamoxifen (TAM) plus UFT for 2 years was compared with TAM alone for 2 years. Samples of tumor tissue were obtained from 192 premenopausal women with node-positive invasive breast cancer. The tissue samples were examined immunohistochemically to study the expression of thymidylate synthase (TS), thymidine phosphorylase (TP), and dihydropyrimidine dehydrogenase (DPD), as well as the expression of HER2 and p53. In patients with TS-positive tumors, the risk of relapse was significantly lower in the tamoxifen plus UFT group than in the tamoxifen alone group. After 2 years, however, there was a trend towards a decrease in the relative predictive value (RPV) of TS with time. No relationship to outcome was detected for TP or DPD. Expression of HER2 or p53 was a significant prognostic indicator in the tamoxifen alone group. TS, but not TP or DPD, may be a useful predictor of response
\end{abstract}

Correspondence to: Dr Masakazu Toi, Department of Surgery (Breast Surgery), Graduate School of Medicine, Kyoto University, 54 Shogoin-Kawaramachi, Sakyo-ku, Kyoto 606-8507, Japan

E-mail: toi@kuhp.kyoto-u.ac.jp

Key words: tegafur-uracil, predictive factor, thymidylate synthase, thymidine phosphorylase, dihydropyrimidine dehydrogenase, breast cancer to UFT therapy. After 2 years, the RPV of TS decreased with time, suggesting that 2 years of treatment with oral fluorouracil derivatives may be inadequate. Further studies are required to investigate this possibility.

\section{Introduction}

UFT is an oral formulation combining tegafur, a prodrug of 5-fluorouracil, with uracil, an inhibitor of dihydropyrimidine dehydrogenase (DPD), the rate-limiting enzyme governing the metabolism of 5-fluorouracil. Recently, many studies have demonstrated that adjuvant treatment with tegafur-uracil (UFT) is effective against lung cancer and other types of solid tumors (1-4). In breast cancer, the therapeutic usefulness of adjuvant chemotherapy with tegafur preparations has been studied in Japan and other countries for more than 20 years $(5,6)$. Recently, Noguchi et al (7) reported the results of a pooled analysis of 6 randomized clinical trials in women with nodenegative breast cancer. Their analysis demonstrated that survival was significantly longer in patients who received UFT than in those who did not. In addition, the effects of combined treatment with UFT and tamoxifen were found to be additive. These findings suggested that UFT may be useful for the management of primary breast cancer, although controlled studies with commonly used regimens for polychemotherapy, such as anthracycline plus cyclophosphamide (AC) and cyclophosphamide plus methotrexate plus fluorouracil (CMF), have yet to be reported.

Recent studies have shown that S-1, a combination of tegafur and 5-chloro-2,4-dihydropyrimidine (CDHP), a more potent inhibitor of DPD than uracil, has high antitumor activity against metastatic breast cancer (8). Other studies with 5fluorouracil derivatives have demonstrated that combined treatment with capecitabine and docetaxel significantly prolongs survival among women with anthracycline-resistant breast cancer, as compared with docetaxel alone (9). Various 
trials are now being performed in preoperative or postoperative settings (10). These drugs will most likely play an important role in the future treatment of breast cancer. The benefits of oral 5-fluorouracil derivatives would be further enhanced by the ability to predict response, thereby identifying patients most likely to benefit from treatment and increasing the benefit-risk ratio.

Various approaches have been proposed to predict the response to oral 5-fluorouracil derivatives. Experimental and clinical evidence has suggested that tumor levels of enzymes involved in nucleoside metabolism, such as thymidylate synthase (TS), thymidine phosphorylase (TP), and dihydropyrimidine dehydrogenase (DPD), may be useful for predicting the response to oral 5-fluorouracil derivatives. Predictive accuracy may be further enhanced by using these enzymes in conjunction with other molecular markers.

We retrospectively examined whether the expression of the 3 enzymes TS, DPD, and TP and that of the oncogene HER2 and the tumor-suppressor gene p53 in breast cancer tissue could be used to predict the response to treatment with tamoxifen plus UFT. Resected tissue specimens were obtained from women with breast cancer who were enrolled in the 3rd Adjuvant Chemo-Endocrine Therapy for Breast Cancer (ACETBC) trials, randomized controlled studies comparing tamoxifen alone with tamoxifen plus UFT after surgery.

\section{Patients and methods}

Combined analysis of three randomized trials. A meta-analysis of 5 randomized controlled trials $(n=1987)$ performed by the ACETBC study group in Japan has shown that the reduction in the risk of recurrence after treatment with UFT was $21 \pm 11 \%$ $(\mathrm{P}=0.06)$ in women with stage I to IIIA breast cancer who underwent mastectomy (5).

Three of these trials examined the effect of adding UFT (300-400 mg/day) to tamoxifen (20-30 mg/day) in women with estrogen-receptor (ER)-positive tumors who postoperatively received adjuvant chemotherapy for 2 years. ER status was determined at each center. Either biochemical (enzyme immunoassay) or immunohistochemical techniques were used. In 2 of these trials, mitomycin $C\left(10 \mathrm{mg} / \mathrm{m}^{2}\right)$ was given intravenously on the day of surgery. Combined analysis of these 3 trials ( $n=1225$; median follow up, 5.7 years) was performed according to the method of Peto (Fig. 1). The reduction in the risk of recurrence after treatment with UFT plus tamoxifen was found to be $26 \pm 12 \%$ ( $\mathrm{p}=0.037$ ). Subset analyses of pooled data in the 3 trials showed that UFT was most effective in premenopausal women with metastases to the axillary lymph nodes (reduction in odds of recurrence, $35 \pm 17 \%$ ). We retrospectively studied the predictive values of biomarkers of response in this patient subset.

\section{Immunohistochemically studied biomarkers}

Collection of tumor samples. A list of subjects was submitted to centers that had agreed to participate in this biomarker study and had registered at least 5 patients to the 3rd ACETBC study. All available paraffin-embedded samples were sent from the centers to the operational office by mail. The samples were stored at room temperature until predictive markers were evaluated.

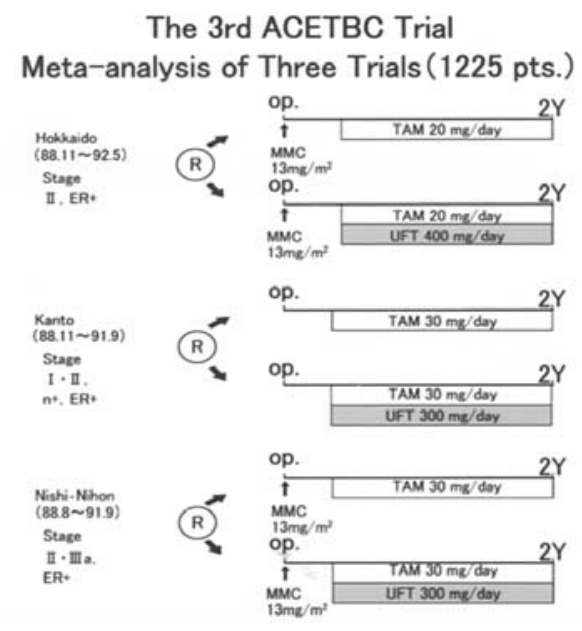

Figure 1. Protocols of the 3rd ACETBC trial.

\section{Immunohistochemical labeling}

Antibodies. TS polyclonal antibody RTSSA (dilution, 1:100; Taiho Pharmaceutical Co., Ltd., Tokyo, Japan), TP monoclonal antibody TMA-1 (dilution, 1:100; Taiho Pharmaceutical Co., Ltd.), DPD polyclonal antibody RDPDPA (dilution, 1:100; Taiho Pharmaceutical Co., Ltd.), HER2 polyclonal antibody A0485 (Dako, Carpinteria, CA, USA; dilution, 1:100), and p53 (DO7) monoclonal antibody (Novo-castra, Newcastle, UK; dilution, 1:40) were used for immunohistochemical analyses.

Immunohistochemical analyses. Immunohistochemical analyses were performed at a single central laboratory using the antibodies described above and mouse IgG (Dako) as negative control. An indirect avidin-biotin-peroxidase method was used. Briefly, deparaffinized tissue sections were treated with $0.3 \%$ hydrogen peroxide in methanol to block endogenous peroxidase activity. After washing with phosphate bufferedsaline (PBS) containing $0.05 \%$ Tween-20, the sections were treated with $1.5 \%$ normal horse serum in PBS and incubated with each of the antibodies or with mouse $\operatorname{IgG}$ for $1 \mathrm{~h}$ at room temperature. The sections were washed again with PBS, incubated with biotinylated anti-mouse $\operatorname{IgG}$ (Dako) for $30 \mathrm{~min}$, washed again with Tween-20-PBS, incubated with an elite $\mathrm{ABC}$ kit (Vector, Burlingame, CA, USA) for $30 \mathrm{~min}$, and visualized with the use of 3,3'-diaminobenzidine tetrahydrochloride-hydrogen peroxide as chromogen. The sections were then counterstained with hematoxylin, dehydrated, and mounted.

Evaluation of staining. The slides were evaluated independently by 3 experienced pathologists (A.F., K.M., T.H.) blinded with regard to treatment group and outcome. Each pathologist evaluated TS, TP, and DPD on the basis of staining intensity of the cytoplasm, scored according to a 4-grade scale (0 to 3 ), and staining rate, also scored according to a 4 -grade scale ( $\leq 25 \%, 0 ;>25 \%$ to $\leq 50 \%, 1 ;>50 \%$ to $\leq 75 \%, 2$; and $>75 \%, 3$ ). The scores agreed on by 2 or more of the pathologists were adopted. Concordance rates of the evaluations among 2 or more pathologists were as follows: TS, staining intensity $95 \%$, staining rate $80 \%$; TP, staining intensity $92 \%$, staining rate 
Table I. Patients' characteristics in the biomarker study.

\begin{tabular}{|c|c|c|c|}
\hline & $\begin{array}{c}\text { TAM group } \\
(n=97)\end{array}$ & $\begin{array}{l}\text { UFT group } \\
\quad(n=95)\end{array}$ & p-value \\
\hline \multicolumn{4}{|l|}{ Age } \\
\hline$\leq 50$ & 89 & 89 & 0.78 \\
\hline$>51$ & 8 & 6 & \\
\hline \multicolumn{4}{|c|}{ Number of nodes involved } \\
\hline $1-3$ & 65 & 73 & 0.15 \\
\hline$\geq 4$ & 32 & 22 & \\
\hline \multicolumn{4}{|l|}{ Tumor size } \\
\hline$<2 \mathrm{~cm}$ & 23 & 24 & 0.87 \\
\hline$\geq 2 \mathrm{~cm}$ & 74 & 71 & \\
\hline \multicolumn{4}{|c|}{ TS expression } \\
\hline Positive & 57 & 48 & 0.31 \\
\hline Negative & 40 & 47 & \\
\hline \multicolumn{4}{|c|}{ TP expression } \\
\hline Positive & 36 & 39 & 0.86 \\
\hline Negative & 61 & 56 & \\
\hline \multicolumn{4}{|c|}{ DPD expression } \\
\hline Positive & 57 & 66 & 0.13 \\
\hline Negative & 40 & 29 & \\
\hline \multicolumn{4}{|c|}{ HER2 expression } \\
\hline Positive & 14 & 14 & 1.00 \\
\hline Negative & 83 & 81 & \\
\hline \multicolumn{4}{|c|}{ p53 expression } \\
\hline Positive & 30 & 33 & 0.85 \\
\hline Negative & 67 & 62 & \\
\hline
\end{tabular}

All patients had estrogen receptor-positive tumors and were premenopausal.

$87 \%$; and DPD, staining intensity $94 \%$, staining rate $89 \%$. The median score was adopted if all 3 pathologists disagreed on the score. Cases were considered positive if the staining intensity was $\geq 2$, and the staining rate was 3 (staining rate, $>75 \%$ ).

HER2 was evaluated on the basis of staining of the membrane, and p53 was evaluated on the basis of staining of nuclei. The results were considered positive if the staining rate was $\geq 1 \%$. The evaluation agreed on by 2 or more pathologists was adopted (concordance rates among the evaluations of the 3 pathologists were as follows: HER2, 89\%; and p53, 72\%).

Statistical analysis. Relapse-free survival was the outcome used to assess treatment efficacy and was defined as the interval elapsed between the date of surgery and the date of documented disease relapse or death. Relapse-free survival was calculated by the Kaplan-Meier method. Differences between groups in Kaplan-Meier estimates of relapse-free survival were evaluated with the log-rank test and generalized Wilcoxon test. Risk

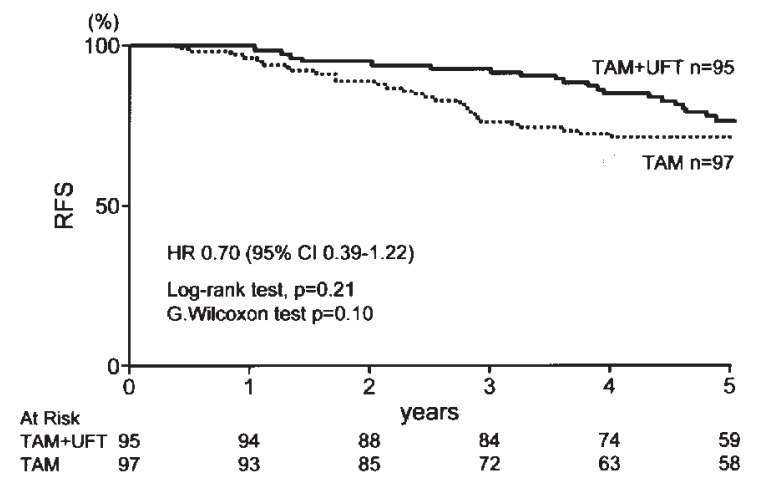

Figure 2. Relapse-free survival (RFS) according to study group ( $\mathrm{n}=192)$.

ratios (RR) were estimated from Cox proportional-hazards regression models. No overall survival analysis was performed in the subgroups of patients identified by the evaluated biological markers because of the small numbers of events in each treatment group. Cox proportional-hazards regression models were also used to test for interactions between biomarkers and treatment.

Relative predictive values (RPV) were determined with use of the following equation, modified from the method described by Hayes (11): RPV for events in the tamoxifen + UFT group was compared with those in the tamoxifen alone group $=\log$ (RR when tumors stained negatively for biomarkers/RR when tumors stained positively for biomarkers). Differences in distributions between groups were compared with the use of the $\chi^{2}$ test. Differences were considered statistically significant when $\mathrm{p}$-values were $<0.05$, and all reported $\mathrm{p}$-values are two-tailed. All analyses were carried out with SAS software (version 6.12).

\section{Results}

Collection of samples. Samples collected from 192 (97 given tamoxifen and 95 given tamoxifen plus UFT) of the 204 women at the centers were assessable. There were no significant differences between the groups in demographic characteristics (age, tumor size, number of lymph node metastases) (Table I). The hazard ratio of the effect of adding UFT to tamoxifen was 0.70 (95\% confidence interval, 0.39 to 1.22$)$ (log-rank test, $\mathrm{p}=0.21$; Wilcoxon test, $\mathrm{p}=0.10$ ) (Fig. 2).

Expression of biomarkers. The rates of positive staining were as follows: TS, 55\% (105/192); TP, 39\% (75/192); DPD, 64\% (123/192); HER2, 15\% (28/192); and p53, 33\% (63/192). The expression rates of these biomarkers were similar in the tamoxifen group and the tamoxifen plus UFT group (Table I).

Relation between relapse-free survival and expression of biomarkers in tumors. Demographic characteristics were similar in women whose tumors stained positively for each biomarker (TS, TP, or DPD) and those whose tumors stained negatively for each biomarker. Univariate analyses showed no significant differences in relapse-free survival between women whose tumors stained positively for TS, TP, or DPD and those whose tumors stained negatively for these 3 
A
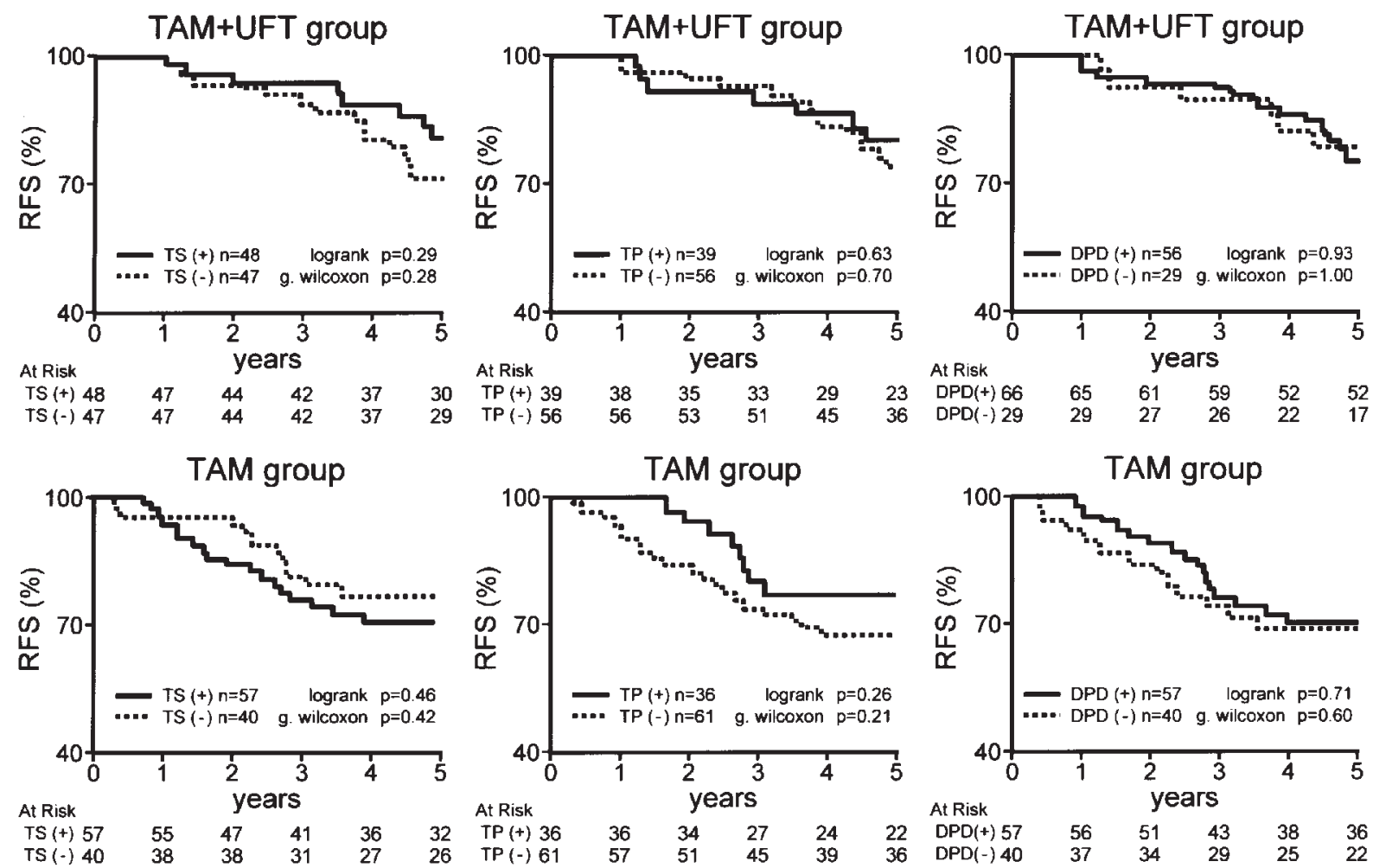

B
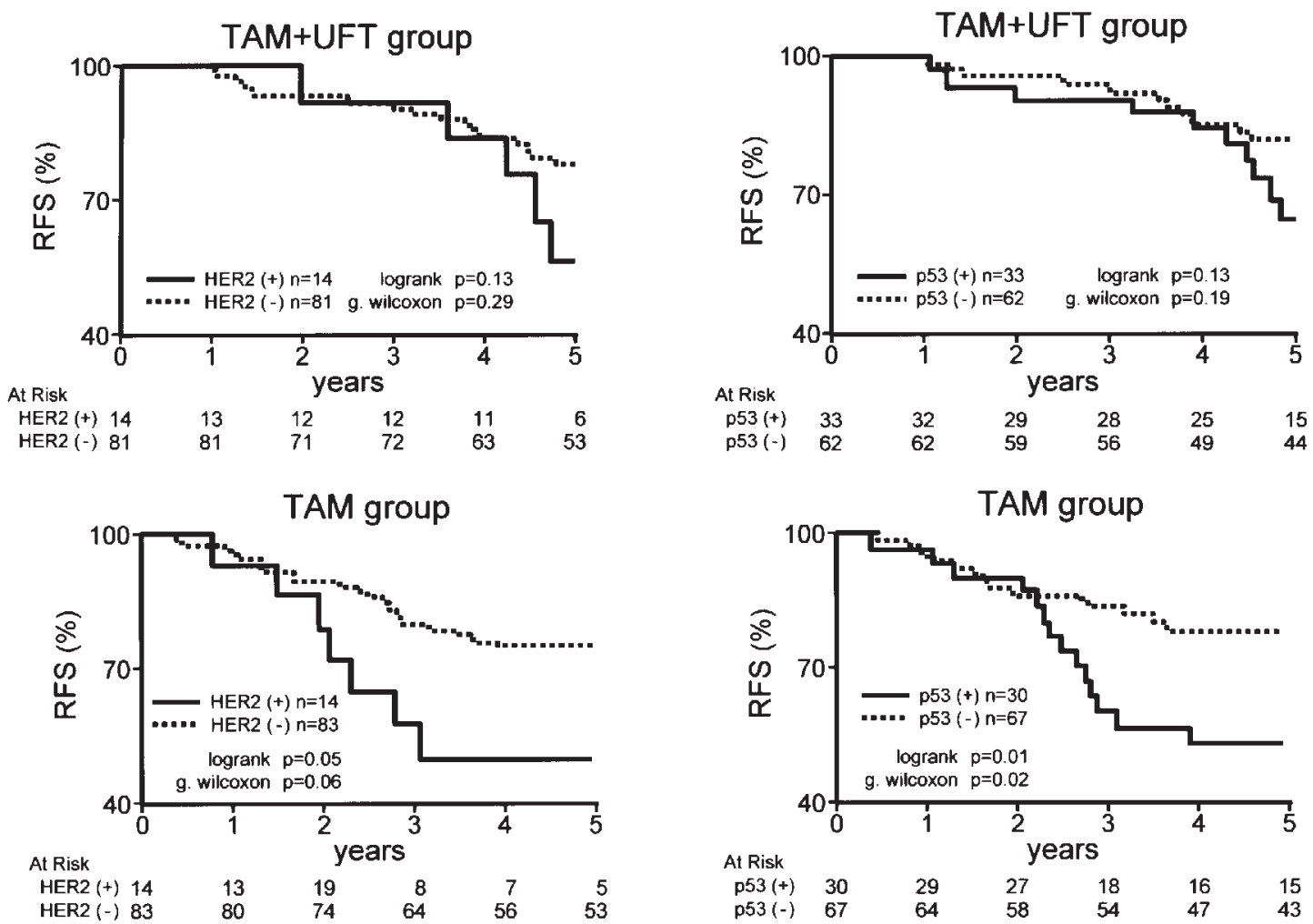

Figure 3. (A) Relation between relapse-free survival (RFS) and tumor expression of thymidylate synthase (TS), thymidine phosphorylase (TP), and dihydropyrimidine dehydrogenase (DPD) according to treatment. (B) Relation between relapse-free survival and tumor expression of HER2 and p53 according to treatment.

biomarkers in either treatment group. Women whose tumors stained positively for HER2 or p53 in the tamoxifen alone group had significantly poorer outcomes than those whose tumors stained negatively for these biomarkers. HER2 and p53 were not significant prognostic factors in the tamoxifen plus UFT group (Fig. 3). 
Table II. Relative risk (TAM+UFT vs. TAM) according to biomarker expression.

\begin{tabular}{|c|c|c|c|c|c|c|c|}
\hline \multirow[t]{2}{*}{ Biomarker } & \multicolumn{3}{|c|}{ Biomarker positive } & \multicolumn{3}{|c|}{ Biomarker negative } & \multirow{2}{*}{$\begin{array}{c}\text { Interaction } \\
\text { p-value }\end{array}$} \\
\hline & $\mathrm{RR}$ & $95 \% \mathrm{CI}$ & $\begin{array}{c}\mathrm{p} \text {-value } \\
\text { (G. Wilcoxon test) }\end{array}$ & $\mathrm{RR}$ & $95 \% \mathrm{CI}$ & $\begin{array}{c}\mathrm{p} \text {-value } \\
\text { (G. Wilcoxon test) }\end{array}$ & \\
\hline TS & 0.48 & $0.20-1.07$ & 0.04 & 1.00 & $0.44-2.36$ & 1.00 & 0.22 \\
\hline TP & 0.80 & $0.28-2.23$ & 0.60 & 0.66 & $0.33-1.30$ & 0.124 & 0.76 \\
\hline DPD & 0.75 & $0.37-1.52$ & 0.29 & 0.61 & $0.21-1.56$ & 0.222 & 0.73 \\
\hline HER2 & 0.59 & $0.17-1.86$ & 0.19 & 0.72 & $0.37-1.37$ & 0.220 & 0.77 \\
\hline p53 & 0.57 & $0.25-1.28$ & 0.09 & 0.78 & $0.35-1.72$ & 0.418 & 0.59 \\
\hline
\end{tabular}

RR, relative risk by addition of UFT to TAM; TS, thymidylate synthase; TP, thymidine phosphorylase; DPD, dihydropyrimidine dehydrogenase.
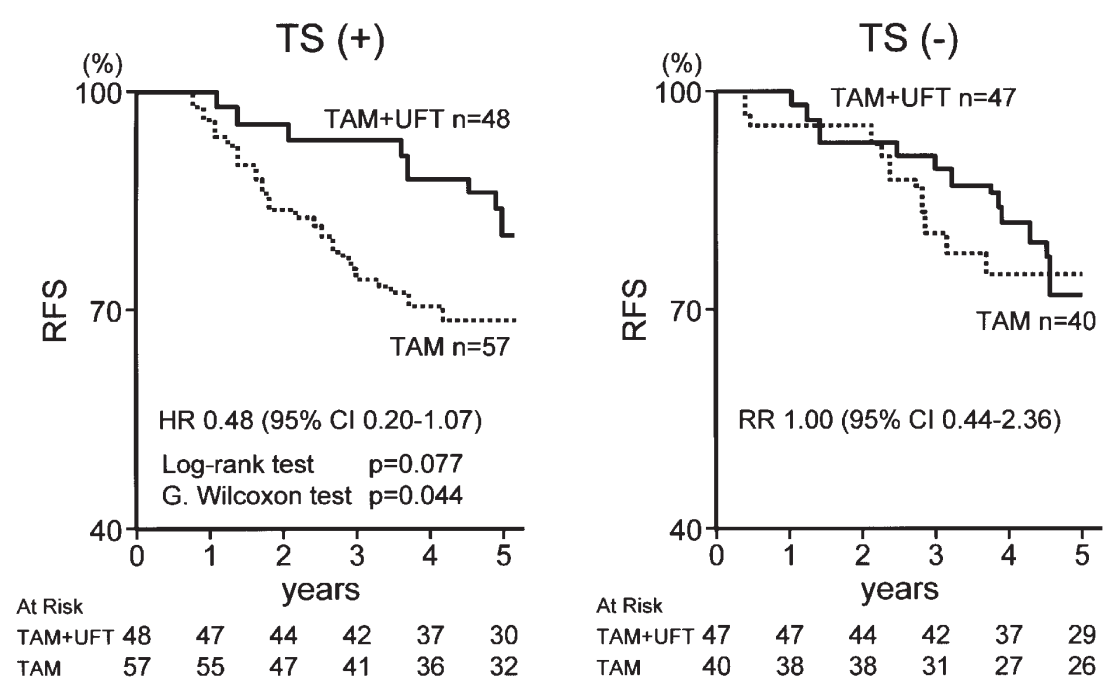

Figure 4. Comparison of relapse-free survival (RFS) between TAM and TAM+UFT treatment according to thymidylate synthase (TS) status.

Relation between expression of biomarkers in tumors and effect of adding UFT to tamoxifen

$T S$. In women with TS-positive tumors, the risk ratio of the effect of adding UFT to tamoxifen was 0.48 (95\% confidence interval, 0.20 to 1.07 ), and response differed significantly between women given tamoxifen alone and those given tamoxifen plus UFT ( $\mathrm{p}=0.04$ by the generalized Wilcoxon test, $\mathrm{p}=0.08$ by the log-rank test). In women with TS-negative tumors, however, there was no significant difference in response (hazard ratio, 1.00; 95\% confidence interval, 0.44 2.36). Interaction testing showed that the expression of TS was not significantly related to the effect of UFT $(\mathrm{p}=0.22)$ (Fig. 3, Table II).

$T P$. The risk ratio of the effect of adding UFT to tamoxifen was 0.80 (95\% confidence interval, $0.28-2.23)$ in women with TP-positive tumors and 0.66 (95\% confidence interval, 0.33-1.30) in women with TP-negative tumors. There were no significant differences in response between the treatment groups. Interaction testing showed no significant relation between the expression of TP and the effect of UFT ( $\mathrm{p}=0.76)$ (Table II).
$D P D$. The risk ratio of the effect of adding UFT to tamoxifen was 0.75 (95\% confidence interval, $0.37-1.52)$ in women with DPD-positive tumors and 0.61 (95\% confidence interval, $0.21-1.56)$ in those with DPD-negative tumors. There were no significant differences between the treatment groups. Interaction testing showed that the expression of DPD was not significantly related to the effect of UFT $(p=0.73)$ (Table II).

HER2. The risk ratio of the effect of adding UFT to tamoxifen was 0.59 (95\% confidence interval, 0.17-1.86) in women with HER2-positive tumors and 0.72 (95\% confidence interval, $0.37-1.37$ ) in those with HER2-negative tumors. There were no significant differences between the treatment groups. Interaction testing showed that the expression of HER2 was not significantly related to the effect of UFT $(p=0.77)$ (Table II).

$p 53$. The hazard ratio of the effect of adding UFT to tamoxifen was 0.57 (95\% confidence interval, 0.25-1.28) in women with $\mathrm{p} 53$-positive tumors and 0.78 (95\% confidence interval, $0.35-1.72$ ) in women with p53-negative tumors. There were no significant differences between the treatment groups. 
A

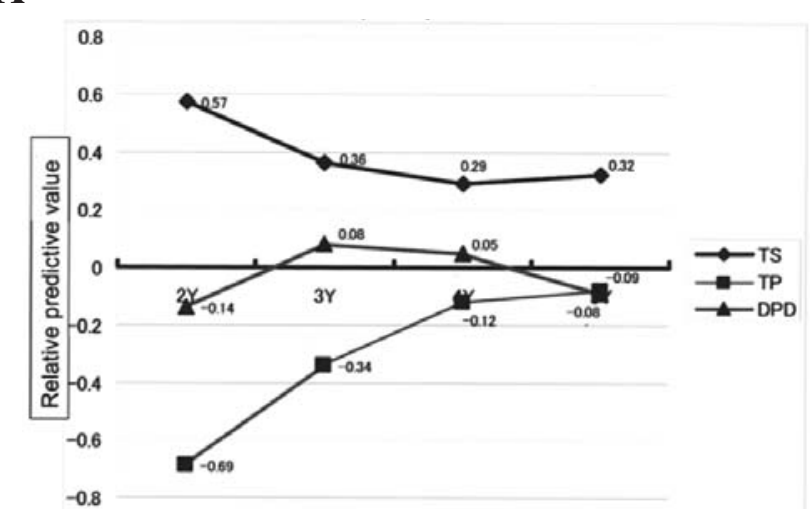

B

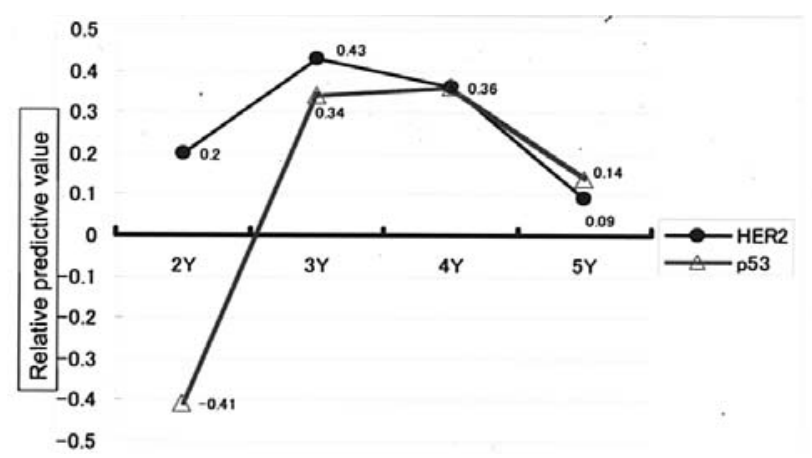

Figure 5. (A) Change in relative predictive values (TS, TP, and DPD). (B) Change in relative predictive values (HER2 and p53). Relative predictive value: $\log [R R$ of marker $(-) / R R$ of marker $(+)]$. RR: risk ratio (TAM vs. TAM+UFT).

Interaction testing demonstrated no relation between the expression of $\mathrm{p} 53$ and the effect of UFT $(\mathrm{p}=0.58)$ (Table II).

Changes in RPV. Changes in the RPV of each biomarker over time are shown in Fig. 5. (The RPV at 1 year could not be determined because some subgroups of patients had no events at 1 year.) The RPV of TS gradually decreased with time for up to 4 years ( 0.57 at 2 years, 0.34 at 3 years, and 0.29 at 4 years), and was 0.32 at 5 years. The absolute value for the RPV of TP gradually decreased over time (-0.67 at 2 years, -0.34 at 3 years, -0.12 at 4 years, and -0.08 at 5 years $)$. The RPV of DPD was approximately 0 for up to 5 years $(-0.14$ at 2 years, 0.08 at 3 years, 0.05 at 4 years, and -0.09 at 5 years). The RPV of HER2 was 0.20 at 2 years, 0.43 at 3 years, 0.36 at 4 years, and 0.09 at 5 years. The RPV of p53 was -0.41 at 2 years, 0.34 at 3 years, 0.36 at 4 years, and 0.14 at 5 years.

\section{Discussion}

We immunohistochemically studied whether the biomarkers TS, TP, DPD, HER2, and p53 could be used to predict the effect of adding UFT to tamoxifen in women with breast cancer who underwent mastectomy. In women with TSpositive tumors, relapse-free survival was significantly better in the tamoxifen plus UFT group than in the tamoxifen group, whereas there was no significant difference between the treatment groups in women with TS-negative tumors. These results suggest that TS can be used to predict the response to
UFT plus tamoxifen, although interaction testing showed no significant interaction between TS expression and treatment response.

Several studies have reported that TS can be used to predict the response to 5-fluorouracil-based adjuvant chemotherapy in patients with colorectal cancer (12-15). These studies consistently found that 5-fluorouracil-based chemotherapy was ineffective for patients with TS-negative tumors, but effective for patients with TS-positive tumors. Pestalozzi et al (16) examined whether TS could be used to predict treatment response in women with breast cancer who were enrolled in a randomized controlled trial (the International Breast Cancer Study Group-V) comparing 1 course of CMF given perioperatively with 6 courses of CMF given postoperatively. Their results showed that suppression of recurrence after 6 courses of postoperative CMF was superior to that after 1 course of perioperative CMF only among women who had TS-positive tumors. Our results are in accordance with their findings. TS, an enzyme involved in DNA synthesis, catalyzes the methylation of deoxyuridine monophosphate to produce deoxythymidine monophosphate. TS is targeted by 5-fluorouracil.

Most experimental studies using cell lines and studies of metastatic cancers (17) have shown that high TS expression is associated with a low antitumor response to 5-fluorouracil, a finding that conflicts with the results of studies in an adjuvant setting. Recent experimental studies by Rahman et al (18) have reported that TS has oncogene-like properties. Overexpression of TS under the condition of serum deprivation was clearly demonstrated to induce apoptosis. Therefore, overexpression of TS due to tumor-related or environmental factors may alter the response to 5-fluorouracil-based chemotherapy. In addition, a recent investigation found that tamoxifen upregulates TS (19). This phenomenon may have a part in the enhanced response to adjuvant chemotherapy with tamoxifen plus UFT.

TP expression was not significantly related to the effect of adding UFT to tamoxifen. TP is an enzyme involved in nucleoside metabolism, antiapoptosis activity, and the promotion of neovascularization. It also converts capecitabine, a prodrug of 5-fluorouracil, and 5'-deoxy-5-fluorouridine (5'-DFUR), an intermediate metabolite of capecitabine, to 5fluorouracil. Many basic and clinical trials have reported the relation between TP expression and the effects of capecitabine and 5'-DFUR (10). Tominaga et al (20) immunohistochemically studied the relation between TP expression and the response to 5'-DFUR in women with early breast cancer who were enrolled in a randomized controlled trial comparing surgery alone with postoperative adjuvant chemotherapy with 5'-DFUR. They concluded that TP expression can be used to predict the response to 5'-DFUR. UFT is a prodrug of 5-fluorouracil, combining tegafur with uracil. Tegafur is converted to 5-fluorouracil principally by liver cytochrome CYP2A6 (21). This mechanism may account for the lack of a relation between TP expression and the effect of adding UFT to tamoxifen in this study.

DPD expression in tumors was also not significantly related to the effect of adding UFT to tamoxifen. DPD, present mainly in the liver, is a rate-limiting enzyme that inactivates 5-fluorouracil. DPD activity in tumors is related 
to sensitivity to 5-fluorouracil. Tumors with high DPD expression are thought to respond poorly to 5-fluorouracil derivatives. Indeed, some studies have reported that sensitivity to capecitabine or doxifluridine is governed by DPD (22-24). UFT contains uracil, an inhibitor of DPD, and may be effective against tumors with high expression levels of DPD (25). This characteristic may account for the fact that the effect of adding UFT to tamoxifen was unrelated to tumor DPD expression.

The expression of HER2 and of p53 was also unrelated to the effect of adding UFT to tamoxifen. Previous studies have reported that the expression of HER2 and p53 is related to the response to anthracycline-based chemotherapy $(26,27)$. However, our study suggests that these factors do not influence the response to UFT. HER2 and p53 were significant prognostic factors in the tamoxifen alone group. Because we did not evaluate these factors in the groups not given tamoxifen, we cannot be certain, but our results suggest that HER2 and p53 are predictive markers of the response to treatment with tamoxifen alone. This notion is supported by the findings of Carlomagno et al (28), who reported that overexpression of HER2 is related to the response to tamoxifen in women with breast cancer.

Hayes described a method for quantifying the pure predictive values of biomarkers for forecasting treatment response (11). He used risk ratio (RR) in a treated group relative to that in a control group for subgroups of patients whose tumors were positive or negative for a given biomarker. The RR was used in the following equation to derive the RPV of the biomarker: RPV $=[1$ - RR (biomarker-positive tumors)]/[1 - RR (biomarker-negative tumors)]. Because $\mathrm{RR}$ was often $>1$ for patients with either biomarker-positive or -negative tumors, we modified Hayes' method and used the following equation: RPV $=\log [\mathrm{RR}$ (biomarker-negative tumors)]/[RR (biomarker-positive tumors)]. The RPV scores were calculated and plotted over time to examine the time course of the RPV (Fig. 4). The RPV was positive if the treatment response was greater when tumors were biomarker positive. Conversely, the RPV was negative if the treatment response was greater when tumors were biomarker negative.

The higher the absolute value of the RPV, the stronger was the power to predict treatment response. Because the natural logarithm was used, the predictive power can be considered weak if the absolute value was $<0.3$ and strong if the absolute value was $\geq 0.5$. The RPV of TS was 0.57 at 2 years and was then gradually decreased with time, but remained at $>0.3$ at 5 years. These data suggest that TS is a pure predictive factor of the response to UFT.

A likely explanation for the reduction in the RPV of TS with time is that the magnitude of the effect of adding UFT to tamoxifen decreased from year 2 onward. A recent overview of randomized trials of adjuvant therapy compiled by the Early Breast Cancer Trialists' Collaborative Group (EBCTCG) (29) showed that the response to poly-chemotherapy, including regimens such as $\mathrm{AC}$ and $\mathrm{CMF}$, diminishes with time, suggesting that this phenomenon is commonly associated with chemotherapy. In the studies analyzed, both tamoxifen and UFT were given for 2 years. Treatment response may persist if UFT is continued for more than 2 years. However, these data should be interpreted with caution because specific subgroups of patients were studied retrospectively.
The RPV of TP was -0.69 at 2 years, and the absolute values were low at 4 and 5 years ( -0.12 and -0.08 , respectively). As mentioned previously, TP was not a statistically significant predictive factor in our study, but there was a trend toward a higher additive effect of UFT when TP was negative. The RPV of DPD consistently remained at approximately 0 , suggesting that the value of DPD for predicting the response to UFT was low.

The RPVs of HER 2 and p53 were $>0.3$ at 3 and 4 years, but neither of these biomarkers were significant predictive factors in our study. This is attributed to the fact that positive rates for HER2 and p53 were low in our study, thereby diminishing statistical power. Interestingly, the time courses of the RPVs of these markers differed from those of TS and TP.

Our results suggested that the expression of TP and DPD, factors related to the response to capecitabine, do not influence the response to UFT. Therefore, different types of oral fluorouracil derivatives may be most effective in distinct subgroups of patients. In the future, expression of TS, DPD, and TP might be useful for selecting patients most likely to respond to tegafur-based oral fluorouracil derivatives, such as UFT and S-1, and those more likely to respond to capecitabine.

At present, however, breast cancer is often treated by a multidisciplinary approach. Care should be exercised when using oral fluorouracil derivatives in combination with other anticancer drugs because the latter may modify nucleosidemetabolizing enzymes, thereby affecting the metabolism of fluorouracil (30). The measurement of biomarkers before and after treatment may also have an important role in the selection of preoperative chemotherapy.

An important limitation of our study was the retrospective design and the inclusion of only a subset of patients (nodepositive premenopausal women) who were enrolled in randomized controlled trials. Our results must therefore be verified in prospective randomized controlled studies in which women with breast cancer are assigned to adjuvant treatment on the basis of the prior determination of biomarker levels.

\section{References}

1. Akasu T, Moriya Y, Ohashi Y, Yoshida S, Shirao K and Kodaira S: Adjuvant chemotherapy with uracil-tegafur for pathological stage III rectal cancer after mesorectal excision with selective lateral pelvic lymphadenectomy: a multicenter randomized controlled trial. Jpn J Clin Oncol 36: 237-244, 2006.

2. Kato H, Ichinose Y, Ohta M, Hata E, Tsubota N, Tada H, et al: A randomized trial of adjuvant chemotherapy with uraciltegafur for adenocarcinoma of the lung. N Engl J Med 350: 1713-1721, 2004.

3. Kato T, Ohashi Y, Nakazato H, Koike A, Saji S, Suzuki H, et al: Efficacy of oral UFT as adjuvant chemotherapy to curative resection of colorectal cancer: multicenter prospective randomized trial. Langenbecks Arch Surg 386: 575-581, 2002.

4. Lembersky BC, Wieand HS, Petrelli NJ, O'Connell MJ, Colangelo LH, Smith RE, et al: Oral uracil and tegafur plus leucovorin compared with intravenous fluorouracil and leucovorin in stage II and III carcinoma of the colon: results from National Surgical Adjuvant Breast and Bowel Project Protocol C-06. J Clin Oncol 24: 2059-2064, 2006.

5. Kasumi F, Yoshimoto M, Uchino J, Abe R, Nomura Y, Sugimachi K, et al: Meta-analysis of five studies on tegafur plus uracil (UFT) as post-operative adjuvant chemotherapy for breast cancer. Oncology 64: 146-153, 2003. 
6. Yoshida M, Abe O, Uchino J, Kikuchi K, Abe R, Enomoto K, et al: Meta-analysis of the second collaborative study of adjuvant chemoendocrine therapy for breast cancer (ACETBC) in patients with stage II, estrogen-receptor-positive breast cancer. Breast Cancer 4: 93-101, 1997.

7. Noguchi S, Koyama H, Uchino J, Abe R, Miura S, Sugimachi K, et al: Postoperative adjuvant therapy with tamoxifen, tegafur plus uracil, or both in women with node-negative breast cancer: a pooled analysis of six randomized controlled trials. J Clin Oncol 23: 2172-2184, 2005.

8. Saeki T, Takashima S, Sano M, Horikoshi N, Miura S, Shimizu S, et al: A phase II study of S-1 in patients with metastatic breast cancer - a Japanese trial by the S-1 Cooperative Study Group, Breast Cancer Working Group. Breast Cancer 11: 194-202, 2004.

9. O'Shaughnessy J, Miles D, Vukelja S, Moiseyenko V, Ayoub JP, Cervantes G, et al: Superior survival with capecitabine plus docetaxel combination therapy in anthracycline-pretreated patients with advanced breast cancer: phase III trial results. J Clin Oncol 20: 2812-2823, 2002.

10. Toi M, Atiqur Rahman M, Bando H and Chow LW: Thymidine phosphorylase (platelet-derived endothelial-cell growth factor) in cancer biology and treatment. Lancet Oncol 6: 158-166, 2005.

11. Hayes DF: Do we need prognostic factors in nodal-negative breast cancer? Arbiter. Eur J Cancer 36: 302-306, 2000.

12. Edler D, Glimelius B, Hallstrom M, Jakobsen A, Johnston PG, Magnusson I, et al: Thymidylate synthase expression in colorectal cancer: a prognostic and predictive marker of benefit from adjuvant fluorouracil-based chemotherapy. J Clin Oncol 20: 1721-1728, 2002.

13. Paradiso A, Simone G, Petroni S, Leone B, Vallejo C, Lacava J, et al: Thymidilate synthase and $\mathrm{p} 53$ primary tumour expression as predictive factors for advanced colorectal cancer patients. $\mathrm{Br}$ J Cancer 82: 560-567, 2000.

14. Takenoue T, Nagawa H, Matsuda K, Fujii S, Nita ME, Hatano K, et al: Relation between thymidylate synthase expression and survival in colon carcinoma, and determination of appropriate application of 5-fluorouracil by immunohistochemical method. Ann Surg Oncol 7: 193-198, 2000.

15. Yamachika T, Nakanishi H, Inada K, Tsukamoto T, Kato T, Fukushima M, et al: A new prognostic factor for colorectal carcinoma, thymidylate synthase, and its therapeutic significance. Cancer 82: 70-77, 1998.

16. Pestalozzi BC, Peterson HF, Gelber RD, Goldhirsch A, Gusterson BA, Trihia $\mathrm{H}$, et al: Prognostic importance of thymidylate synthase expression in early breast cancer. J Clin Oncol 15: 1923-1931, 1997.

17. Adlard JW, Richman SD, Seymour MT and Quirke P: Prediction of the response of colorectal cancer to systemic therapy. Lancet Oncol 3: 75-82, 2002.

18. Rahman L, Voeller D, Rahman M, Lipkowitz S, Allegra C, Barrett JC, et al: Thymidylate synthase as an oncogene: a novel role for an essential DNA synthesis enzyme. Cancer Cell 5: 341-351, 2004.
19. Xie W, Duan R, Chen I, Samudio I and Safe S: Transcriptional activation of thymidylate synthase by 17beta-estradiol in MCF-7 human breast cancer cells. Endocrinology 141: 2439-2449, 2000.

20. Tominaga T, Toi M, Ohashi Y and Abe O: Prognostic and predictive value of thymidine phosphorylase activity in earlystage breast cancer patients. Clin Breast Cancer 3: 55-64, 2002 .

21. Ikeda K, Yoshisue K, Matsushima E, Nagayama S, Kobayashi K, Tyson CA, et al: Bioactivation of tegafur to 5-fluorouracil is catalyzed by cytochrome P-450 2A6 in human liver microsomes in vitro. Clin Cancer Res 6: 4409-4415, 2000.

22. Ishikawa T, Sekiguchi F, Fukase Y, Sawada N and Ishitsuka H: Positive correlation between the efficacy of capecitabine and doxifluridine and the ratio of thymidine phosphorylase to dihydropyrimidine dehydrogenase activities in tumors in human cancer xenografts. Cancer Res 58: 685-690, 1998.

23. Takechi T, Okabe H, Ikeda K, Fujioka A, Nakagawa F, Ohshimo $\mathrm{H}$, et al: Correlations between antitumor activities of fluoropyrimidines and DPD activity in lung tumor xenografts. Oncol Rep 14: 33-39, 2005.

24. Terashima M, Fujiwara H, Takagane A, Abe K, Araya M, Irinoda $\mathrm{T}$, et al: Role of thymidine phosphorylase and dihydropyrimidine dehydrogenase in tumour progression and sensitivity to doxifluridine in gastric cancer patients. Eur J Cancer 38: 2375-2381, 2002.

25. Takechi T, Uchida J, Fujioka A and Fukushima M: Enhancing 5-fluorouracil cytotoxicity by inhibiting dihydropyrimidine dehydrogenase activity with uracil in human tumour cells. Int J Oncol 11: 1041-1044, 1997.

26. Dressler LG, Berry DA, Broadwater G, Cowan D, Cox K, Griffin S, et al: Comparison of HER2 status by fluorescence in situ hybridization and immunohistochemistry to predict benefit from dose escalation of adjuvant doxorubicin-based therapy in node-positive breast cancer patients. J Clin Oncol 23: 4287-4297, 2005.

27. Yamauchi H, Stearns V and Hayes DF: When is a tumor marker ready for prime time? A case study of c-erbB-2 as a predictive factor in breast cancer. J Clin Oncol 19: 2334-2356, 2001.

28. Carlomagno C, Perrone F, Gallo C, De Laurentiis M, Lauria R, Morabito A, et al: c-erb B2 overexpression decreases the benefit of adjuvant tamoxifen in early-stage breast cancer without axillary lymph node metastases. J Clin Oncol 14: 2702-2708, 1996.

29. EBCTCG: Effects of chemotherapy and hormonal therapy for early breast cancer on recurrence and 15-year survival: an overview of the randomised trials. Lancet 365: 1687-1717, 2005.

30. Toi M, Bando H, Horiguchi S, Takada M, Kataoka A, Ueno T, et al: Modulation of thymidine phosphorylase by neoadjuvant chemotherapy in primary breast cancer. Br J Cancer 90: 2338-2343, 2004 